\title{
Blind digital holographic microscopy
}

\author{
Patrick N. Anderson ${ }^{\mathrm{a}}$, Florian Wiegandt ${ }^{\mathrm{a}}$, Daniel J. Treacher ${ }^{\mathrm{a}}$, Matthias M. Mang ${ }^{\mathrm{a}}$, Ilaria \\ Gianani $^{\mathrm{a}}$, Andrea Schiavi ${ }^{\mathrm{a}}$, David T. Lloyd ${ }^{\mathrm{a}}$, Kevin O’Keeffe ${ }^{\mathrm{a}, \mathrm{b}}$, Simon M. Hooker ${ }^{\mathrm{a}}$, and Ian A. \\ Walmsley $^{\mathrm{a}}$ \\ ${ }^{a}$ Clarendon Laboratory, University of Oxford, Parks Road, Oxford, OX1 3PU, UK \\ ${ }^{\mathrm{b}}$ Department of Physics, Swansea University, Singleton Park, Swansea, SA2 8PP, UK
}

\begin{abstract}
A blind variant of digital holographic microscopy is presented that removes the requirement for a well-characterized, highly divergent reference beam. This is achieved by adopting an off-axis recording geometry where a sequence of holograms is recorded as the reference is tilted, and an iterative algorithm that estimates the amplitudes and phases of both beams while simultaneously enhancing the numerical aperture. Numerical simulations have demonstrated the accuracy and robustness of this approach when applied to the coherent imaging problem.
\end{abstract}

Keywords: Digital holography, Phase retrieval

\section{COPYRIGHT NOTICE}

Copyright 2017 Society of Photo-Optical Instrumentation Engineers (SPIE). One print or electronic copy may be made for personal use only. Systematic reproduction and distribution, duplication of any material in this paper for a fee or for commercial purposes, or modification of the content of the paper are prohibited.

Please cite as: Patrick N. Anderson, Florian Wiegandt, Daniel J. Treacher, Matthias M. Mang, Ilaria Gianani, Andrea Schiavi, David T. Lloyd, Kevin O'Keeffe, Simon M. Hooker, Ian A. Walmsley, "Blind digital holographic microscopy", Proc. SPIE 10127, Practical Holography XXXI: Materials and Applications, 101270H (6 April 2017); doi: $10.1117 / 12.2250317$

\section{INTRODUCTION}

Simple optical microscopes provide access to a magnified intensity distribution of strongly scattering and absorbing samples. Problems arise when studying thin, weakly scattering samples samples (including living cells) with such instruments due to a lack of contrast. Coherent imaging solves this problem by measuring both the amplitude and phase distribution of the illuminating beam. Encoded in this phase information are the local thicknesses and refractive indices of the sample. ${ }^{1-3}$ Coherent imaging also has the advantage that it is lensless. This enhances image quality by eliminating optical aberrations and enables the technique to be implemented at exotic wavelengths where optics are unavailable, including the lucrative X-ray region. ${ }^{4}$

In general, there are two popular approaches to coherent imaging and we will provide a modern example of each. The first are holographic techniques, and in particular digital holographic microscopy (DHM). ${ }^{5}$ Here, a probe beam is scattered from a sample and subsequently interfered with a spherical reference beam at the detector. It is then possible to retrieve the amplitudes and phases of the probe beam from the resulting hologram provided the reference is well-known. The second family of techniques rely on iterative algorithms alone to retrieve the amplitudes and phases from intensity measurements of the scattered probe. ${ }^{6}$ One particularly successful method of this type is ptychography. ${ }^{7}$ Here, small translations are applied to the sample and a scattering image is recorded at each position providing robust algorithm convergence when compared to other non-referenced schemes.

Here, we describe a hybrid of these techniques where an iterative algorithm is applied to holographic retrieval. This approach relaxes the requirement for the reference beam to be a well-known spherical wave (similar to Ref. 8 ), while enhancing the effective numerical aperture $\left(\mathrm{NA}_{\text {eff }}\right)$. In Section 3 we will describe the holographic recording geometry and retrieval procedure. Then in Section 4 we will demonstrate the technique via numerical simulations before presenting our conclusions in Section 5.

Further author information: Send correspondence to ian.walmsley@physics.ox.ac.uk 


\section{CONCEPT}

The geometry of interest consists of probe and reference beams that are separated spatially at some effective source plane. A sample (placed in the probe beam) and digital camera are positioned downstream of this plane at $d_{1}$ and $d_{2}$ respectively. This is largely consistent with conventional off-axis DHM, with the key exception that the reference need not be a known or highly divergent. This is accomplished by an iterative retrieval algorithm that accurately estimates the amplitudes and phases of both beams from a sequence of $N$ holograms recorded as the reference is tilted about the source plane. The $k^{\text {th }}$ hologram in such a sequence takes the form

$$
H_{k}=\left|\mathcal{E}_{\text {probe }}\right|^{2}+\left|\mathcal{E}_{\text {ref }}\left(r-\Gamma_{k}\right) \mathcal{A}\left(\Gamma_{k}\right)\right|^{2}+2 \operatorname{Re}\left[\mathcal{E}_{\text {probe }} \mathcal{E}_{\text {ref }}^{*}\left(r-\Gamma_{k}\right) \mathcal{A}^{*}\left(\Gamma_{k}\right)\right],
$$

where $\mathcal{E}_{\text {probe }}$ and $\mathcal{E}_{\text {ref }}$ are the complex electric fields of the probe and reference. In writing Eq. 1 the tilt applied to the reference has been interpreted as a lateral shift $\Gamma$ via the introduction of a shift-dependent complex ancillary field $\mathcal{A}$.

The third term in Eq. 1 provides access to the amplitudes and phases of all three fields. This encoded information $S_{k}$ is straightforward to extract from an experimentally recorded hologram by applying a bandpass filter in the Fourier domain and isolating the sideband. In contrast to conventional holography where the reference is known, robust methods for separating $S_{k}$ into its constituent fields are far more intensive. Here we utilize Mutual Interferometric Characterization of two Electric-fields $(\mathrm{MICE})^{9}$ for this task as it is ideally suited to our geometry. ${ }^{10}$ Following Ref. 9 the error between $S_{k}$ and the third term in Eq. 1 is minimized with respect to each of the three fields yielding the following equations

$$
\begin{aligned}
\mathcal{E}_{\text {probe }} & =\frac{\sum_{k=1}^{K} S_{k}\left(r-\Gamma_{k}\right) \mathcal{E}_{\text {ref }}^{*}\left(r-\Gamma_{k}\right) \mathcal{A}\left(\Gamma_{k}\right)}{\sum_{k=1}^{K}\left|\mathcal{E}_{\text {ref }}\left(r-\Gamma_{k}\right) \mathcal{A}\left(\Gamma_{k}\right)\right|^{2}} \\
\mathcal{E}_{\text {ref }}^{*} & =\frac{\sum_{k=1}^{K} S_{k}\left(r+\Gamma_{k}\right) \mathcal{E}_{\text {probe }}^{*}\left(r+\Gamma_{k}\right) \mathcal{A}\left(\Gamma_{k}\right)}{\sum_{k=1}^{K}\left|\mathcal{E}_{\text {probe }}\left(r+\Gamma_{k}\right) \mathcal{A}\left(\Gamma_{k}\right)\right|^{2}} \\
\mathcal{A}^{*}(\Gamma) & =\frac{\sum S_{k}\left(r-\Gamma_{k}\right) \mathcal{E}_{\text {probe }}^{*} \mathcal{E}_{\text {ref }}\left(r-\Gamma_{k}\right)}{\sum\left|\mathcal{E}_{\text {probe }} \mathcal{E}_{\text {ref }}\left(r-\Gamma_{k}\right)\right|^{2}},
\end{aligned}
$$

where the summation when evaluating $\mathcal{A}^{*}(\Gamma)$ extends over all $\mathrm{k}$ and all elements. Solving Eq. 2 iteratively from a random starting guess for $\mathcal{E}_{\text {ref }}$ and $\mathcal{A}$ has been shown to retrieve the three fields after as few as 10 iterations, with only trivial ambiguities. ${ }^{9}$

In the process of writing Eq. 1 a spherical phase component with radius of curvature $d_{2}$ is removed from each beam. As a result the phases of $\mathcal{E}_{\text {probe }}$ and $\mathcal{E}_{\text {ref }}$ retrieved via Eq. 2 describe the deviation from this spherical wave. This phase would be straightforward to re-insert, however it is useful to utilize this as a means of achieving magnification. For a sample positioned at $d_{1}$, back-propagation over an effective distance

$$
d_{\mathrm{eff}}=\frac{d_{2}^{2}-d_{2} d_{1}}{d_{1}}
$$

results in a magnification

$$
M=\left|\frac{d_{2}}{d_{1}}\right| .
$$

As our technique removes the requirement for a well-known reference beam, provides access to an arbitrarily large $\mathrm{NA}_{\text {eff }}$ by tilting the reference (a similar approach to that used in Ref. 11), and preserves the magnification protocol from conventional DHM we will refer to our approach as blind-DHM.

\section{NUMERICAL SIMULATIONS}

Fig. 1 demonstrates the blind-DHM technique using simulated data. First, a super-Gaussian probe beam with a $200 \mu \mathrm{m}$ waist and flat phase is generated at the source plane. This is propagated $3 \mathrm{~mm}$ to the sample plane 

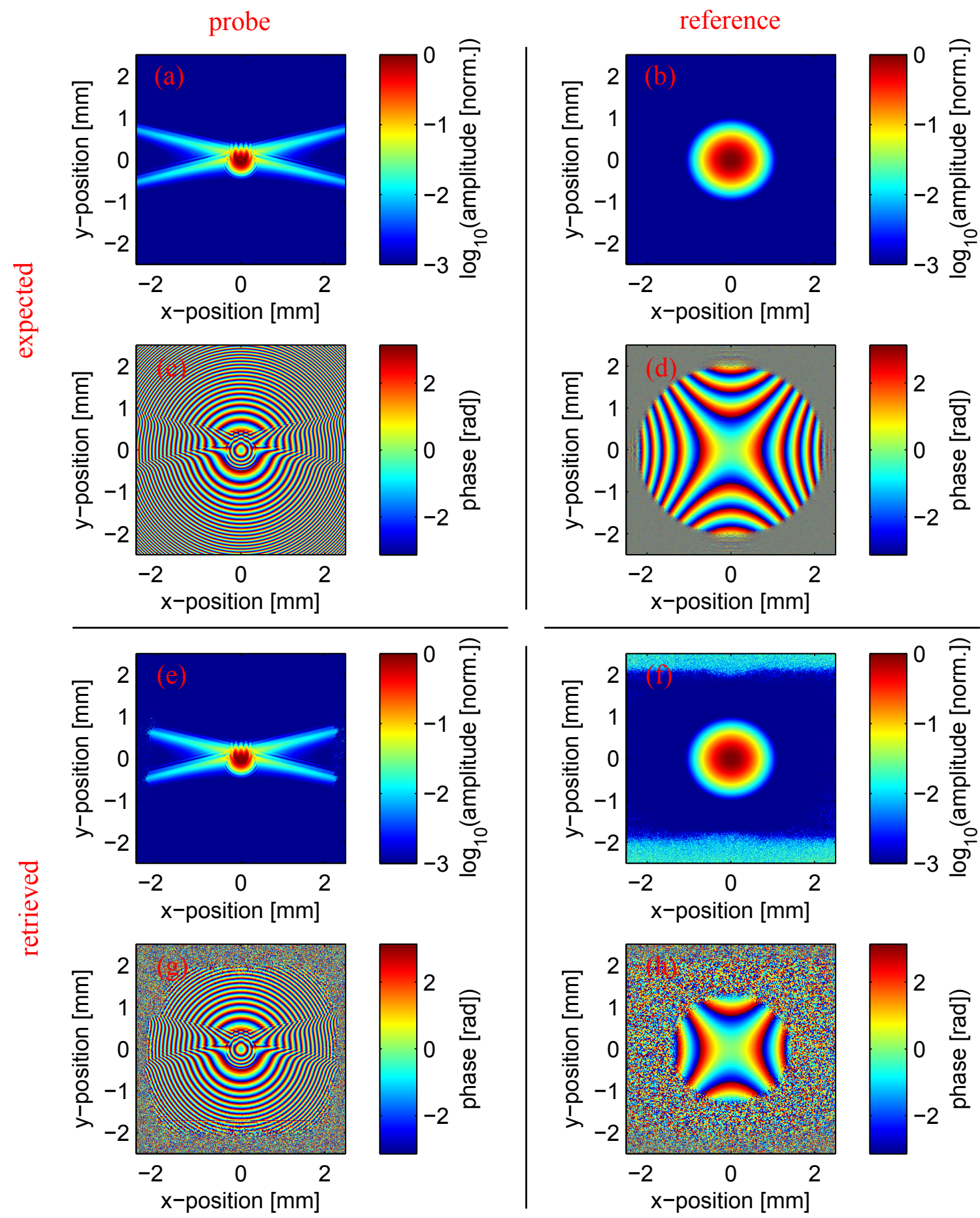

Figure 1. The amplitudes and phases of the scattered probe and astigmatic reference beam at the detector. The expected values are shown in (a-d) and those retrieved via blind-DHM are given in (e-h). Trivial phase ambiguities have been corrected and the logarithm of absolute normalized amplitude is plotted to aid visualization and comparison. See Section 4 for a complete description of the methods and parameters used to simulate the sequence of holograms and perform the retrieval. 

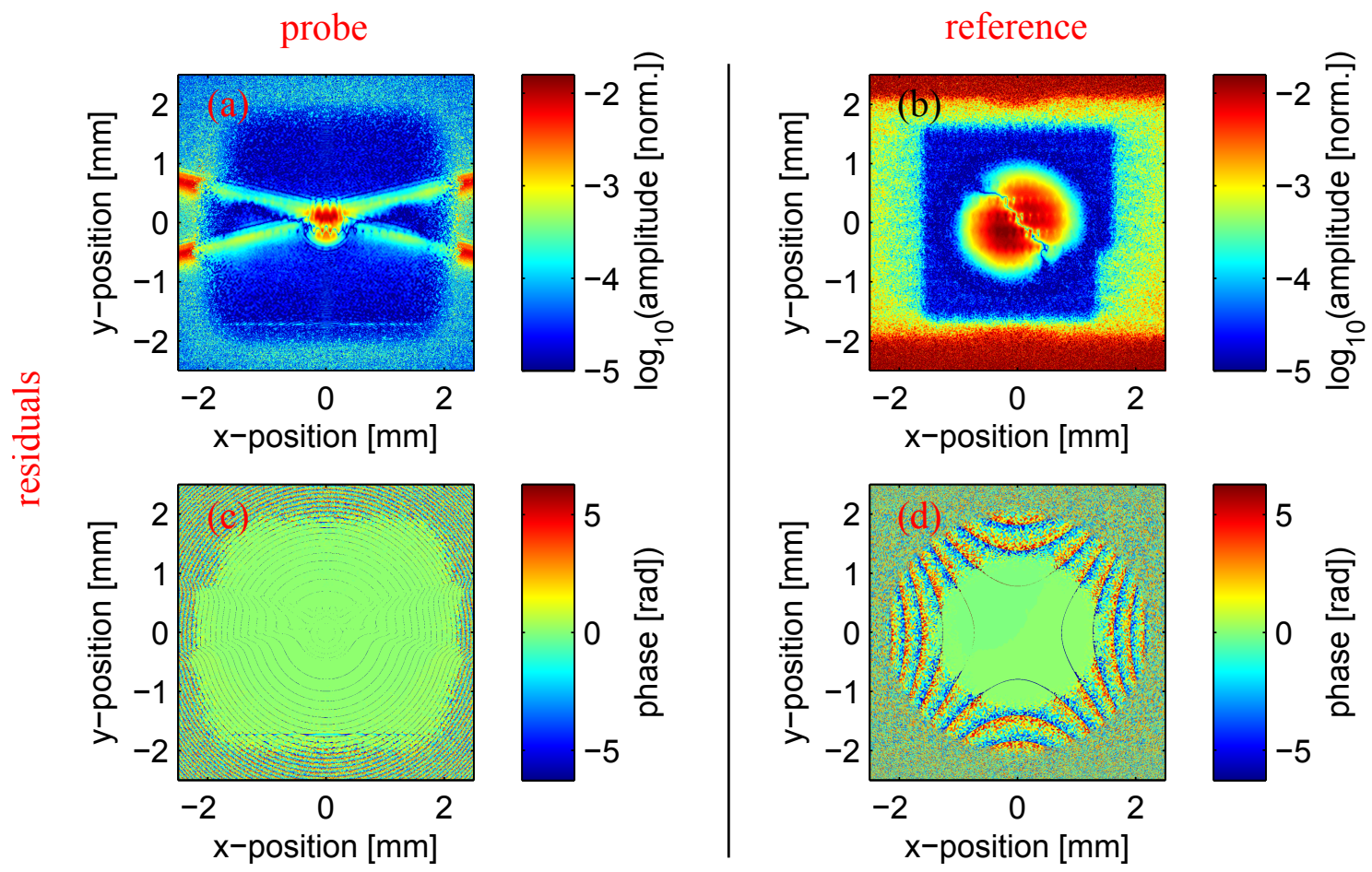

Figure 2. The residuals of the expected and retrieved fields from the simulation in Fig. 1. To visualize the amplitude residuals over a large dynamic range the logarithm of the absolute values are plotted. Note the low phase error over the entire $\mathrm{NA}_{\mathrm{eff}}$ in (c).

where it intercepts a positive triangular binary sample, and then an additional $27 \mathrm{~mm}$ to the detector plane. All numerical propagation is performed using the angular spectrum method (ASM). Next, an astigmatic reference beam is simulated by propagating a Gaussian beam with a $2 \mathrm{~mm}$ waist and flat phase through an $\mathrm{f}_{\mathrm{x}}=74 \mathrm{~mm}$ and $\mathrm{f}_{\mathrm{y}}=76 \mathrm{~mm}$ cylindrical lens system. The reference is aligned such that its circle of least confusion is positioned $937.5 \mu \mathrm{m}$ above the probe beam at the source plane. Finally, the reference beam is propagated $30 \mathrm{~mm}$ to the detector plane. The expected amplitudes and phases of the probe Fig. 1 (a, c) and reference Fig. 1 (b, d) at the detector are plotted. The resulting hologram is mapped onto a detector with $2048 \times 20485 \mu \mathrm{m}$ pixels, each with a 16-bit dynamic range, and random additive noise applied at a signal-to-noise ratio of $40 \mathrm{~dB}$. The peak amplitudes of the beams are equalized at the detector plane and the full dynamic range is utilized with no saturation. The reference beam is then tilted about the source plane so it follows an $11 \times 11$ raster scan pattern across the detector. Subsequent exposures are positioned so $\mathrm{NA}_{\text {eff }}$ is maximized (0.0277 to 0.0737) while maintaining robust algorithm performance. The overlap $O$ between neighboring exposures is key to the success of blind-DHM and its optimization (to a value of $O=0.6539$ ) is discussed in Appendix A.

Due to the scale of the data, finding an iterative solution to Eq. 2 requires non-sequential programming and substantial computational resources. Our particular routine is implemented in $\mathrm{C}++$ and complied using Intel ${ }^{\circledR}$ $\mathrm{C}++$ 15.0.6. Open MPI 1.8.4, Intel ${ }^{\circledR}$ MKL 11.2.4 and Eigen 3.3.10 are utilized for parallelization, fast Fourier transforms, and matrix operations respectively. The retrieval shown in Fig. 1 (e-h) was performed on a machine with 2 8-core Intel ${ }^{\circledR}$ E5-2640V3 processors and 64 GB RAM, and algorithm convergence was achieved after 10 iterations. The algorithm was allowed to run for a further 40 iterations to guarantee convergence. The total run-time was $454.8 \mathrm{~s}$ of which $41.2 \mathrm{~s}$ was associated with input/output, leaving $8.3 \mathrm{~s}$ for each iterative solution of Eq. 2. To aid visualization all trivial phase ambiguities have been removed from Fig. 1 (c-d), and amplitudes have been normalized. This post-processing enables the logarithm of the absolute value of the residuals to be calculated and these are displayed in Fig. 2. Analyzing these plots highlights the high quality estimation of both 

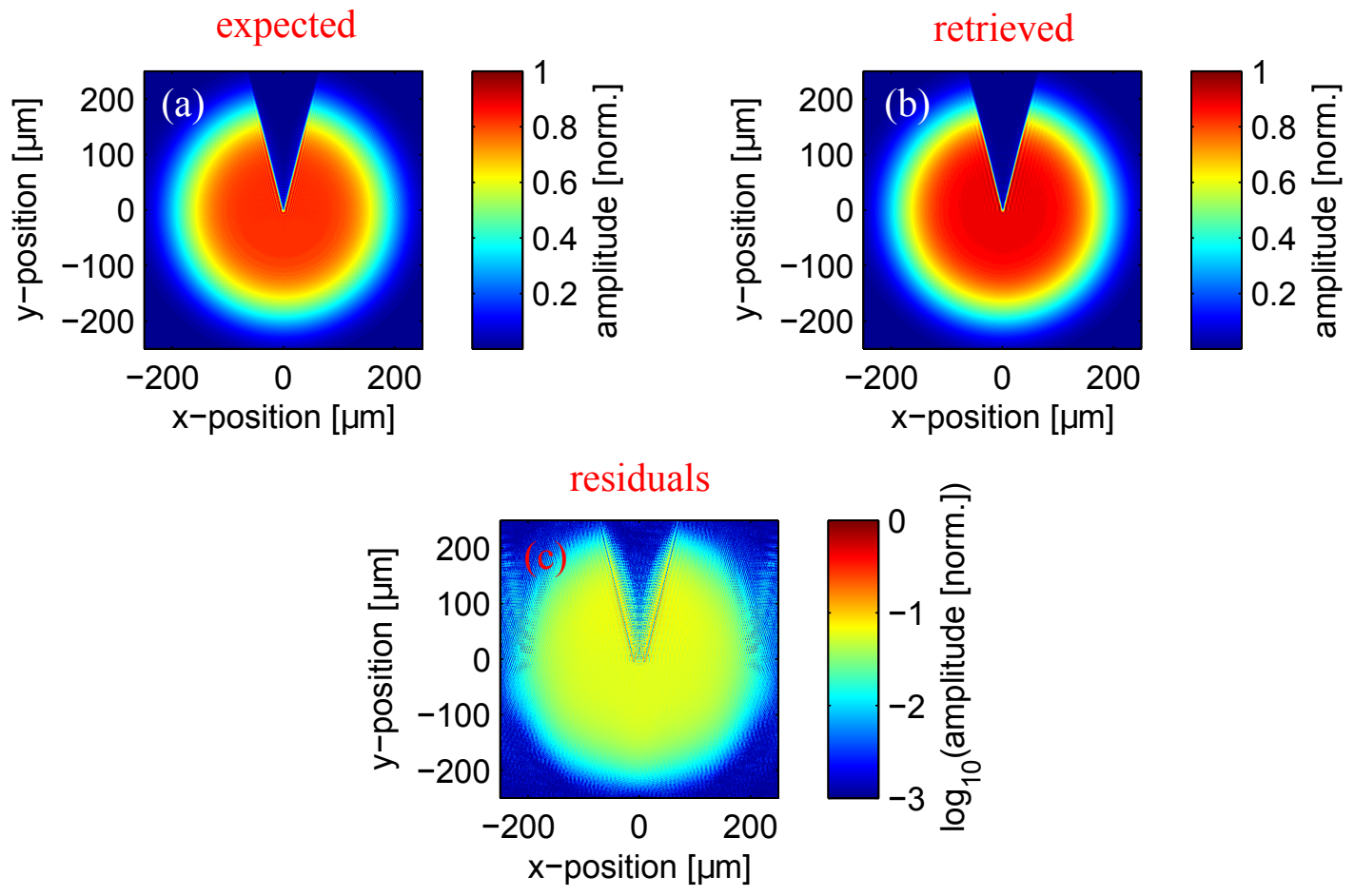

Figure 3. The expected (a) and retrieved (b) normalized amplitudes after back-propagating the fields a distance $d_{\text {eff }}$ to the sample plane. Again, the logarithm of the absolute amplitude residuals are plotted in (c). The 0.1-0.9 spatial edge resolution is estimated to be $4 \mu \mathrm{m}$ from (b). Here, this is limited by $\mathrm{NA}_{\text {eff }}$ which can be increased by recording additional holograms, thus providing a route to diffraction-limited coherent imaging in an entirely lensless geometry.

amplitude and phase within the effective numerical aperture. The transition beyond this region is clearly visible in Fig. 1 (c) where the phase error increases abruptly.

Back-propagating the probe field over a distance $d_{\text {eff }}$ yields a magnified coherent image of the sample. The normalized amplitudes of which are shown in Fig. 3 (b). For comparison the expected values are displayed in 3 (a) and the logarithm of the absolute value of the residuals is once again plotted in 3 (c). The 0.1-0.9 spatial edge resolution can be estimated from Fig. 3 (b) as $4 \mu \mathrm{m}$ which compares favorably to the maximum spatial resolution of $4.3 \mu \mathrm{m}$ imposed by $\mathrm{NA}_{\text {eff. }}$ The small discrepancy is due to a conservative estimate of $\mathrm{NA}_{\text {eff. }}$.

\section{CONCLUSION}

We have presented a blind variant of DHM that alleviates the need for a well-known and highly divergent reference beam. An off-axis DHM recording geometry is adopted where a sequence of holograms is recorded as the reference is tilted about an effective source plane. From this, an iterative algorithm estimates the amplitudes and phases of both beams while simultaneously enhancing the numerical aperture. Numerical simulations have demonstrated the accuracy and robustness of this approach, and a coherent image of a sample has been retrieved with a

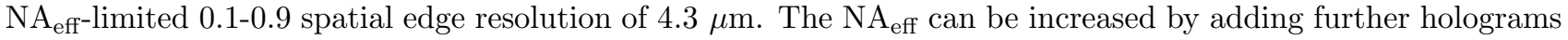
to the sequence, providing a route to diffraction-limited coherent imaging in an entirely lensless geometry.

\section{APPENDIX A. OVERLAP OPTIMIZATION}

The extent to which the spatial profile of the reference overlaps between neighboring holograms is of vital importance to blind-DHM. Fig. 4 shows the retrieved (normalized) amplitudes in the sample plane for a series of overlap parameters between $O=1 \mathrm{Fig} .4$ (a) and $O=0.4$ Fig. 4 (g) in steps of 0.1. 4 (a) is analogous to 

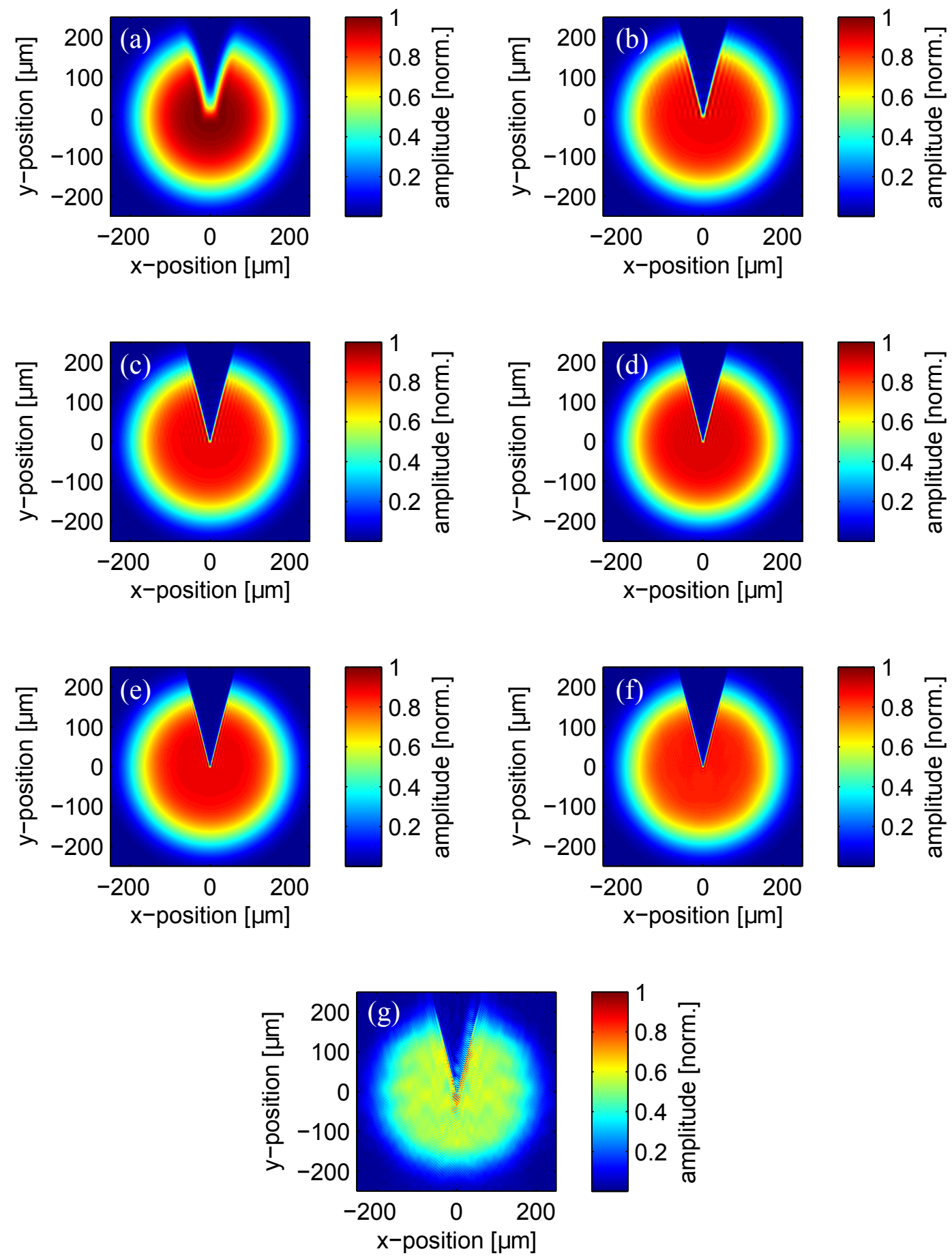

Figure 4. Optimization of the reference overlap parameter $O$ between neighboring holograms. (a - g) Show the retrieved amplitudes in the object plane for $O=1$ to $O=0.4$ with $\Delta O=0.1$. The RMS error is minimum at $O=0.6539$ yielding an $\mathrm{NA}_{\text {eff }}$ of 0.0737 and a maximum spatial resolution of $4.3 \mu \mathrm{m}$. 
for accurate retrieval of the two beams. To identify an intermediary between these regimes the RMS error is minimized in the sample plane, resulting in an optimal value of $O=0.6539$. This corresponds to an $\mathrm{NA}_{\text {eff }}$ of 0.0737 and a maximum spatial resolution of $4.3 \mu \mathrm{m}$.

\section{ACKNOWLEDGMENTS}

This work has been supported by the Engineering and Physical Sciences Research Council (EPSRC) under grant EP/L015137/1 and the European Unions Horizon 2020 research and innovation programme under the Marie Sklodowska-Curie grant agreement No. 641272. The authors would like to acknowledge the use of the University of Oxford Advanced Research Computing (ARC) facility in carrying out this work.

\section{REFERENCES}

[1] Cuche, E., Bevilacqua, F., and Depeursinge, C., "Digital holography for quantitative phase-contrast imaging," Optics Letters 24(5), 291 (1999).

[2] Ou, X., Horstmeyer, R., Yang, C., and Zheng, G., "Quantitative phase imaging via Fourier ptychographic microscopy," Optics Letters 38(22), 4845 (2013).

[3] Mir, M., Bhaduri, B., Wang, R., Zhu, R., and Popescu, G., "Quantitative phase imaging," Progress in Optics 57, 133 (2012).

[4] Chapman, H. N. and Nugent, K. A., "Coherent lensless X-ray imaging," Nature Photonics 4, 833 (2010).

[5] Schnars, U. and Jüptner, W. P. O., "Digital recording and numerical reconstruction of holograms," Measurement Science and Technology 13(9) (2002).

[6] Fienup, J. R., "Phase retrieval algorithms: a comparison," Applied Optics 21(15), 2758 (1982).

[7] Rodenburg, J. M., "Ptychography and Related Diffractive Imaging Methods," Advances in Imaging and Electron Physics 150, 87 (2008).

[8] Raz, O., Leshem, B., Miao, J., Nadler, B., Oron, D., and Dudovich, N., "Direct phase retrieval in double blind Fourier holography," Optics Express 22(21), 24935 (2014).

[9] Bourassin-Bouchet, C., Mang, M. M., Gianani, I., and Walmsley, I. A., "Mutual interferometric characterization of a pair of independent electric fields.," Optics Letters 38(24), 5299 (2013).

[10] Mang, M. M., Bourassin-Bouchet, C., and Walmsley, I. a., "Simultaneous spatial characterization of two independent sources of high harmonic radiation," Optics Letters 39(21), 6142 (2014).

[11] Mico, V., Zalevsky, Z., and García-Martínez, Pascuala García, J., "Synthetic aperture superresolution with multiple off-axis holograms," Journal of the Optical Society of America A 23(12), 3162 (2006). 